\title{
EXTENDED MASS RELATION FOR SEVEN FUNDAMENTAL MASSES AND NEW EVIDENCE OF LARGE NUMBERS HYPOTHESIS
}

\author{
${ }^{1}$ Curtis J. Forsythe and ${ }^{2}$ Dimitar T. Valev \\ ${ }^{1}$ Retired, 21204 East 173rd Street, Pleasant Hill, Missouri 64080, USA \\ ${ }^{2}$ Space Research and Technology Institute, \\ Bulgarian Academy of Sciences, 6000 Stara Zagora, Bulgaria
}

Received 2014-06-23; Revised 2014-07-15; Accepted 2014-07-16

\begin{abstract}
A previously derived mass relation has been extended to seven equidistant fundamental masses covering an extremely large mass range from $\sim 10^{-69}$ to $\sim 10^{53} \mathrm{~kg}$. Six of these masses are successfully identified as mass of the observable universe, Eddington mass limit of the most massive stars, mass of hypothetical quantum "Gravity Atom" whose gravitational potential is equal to electrostatic potential $e^{2} / S$, Planck mass, Hubble mass and mass dimension constant relating masses of stable particles with coupling constants of fundamental interactions. The seventh mass, $\sim 10^{-48} \mathrm{~kg}$ remains unidentified and could be considered as a prediction of the suggested mass relation for an unknown fundamental mass, potentially a yet unobserved light particle. First triad of these masses describes macro objects, the other three masses belong to particle physics masses and the Planck mass appears intermediate in relation to these two groups. Additionally, new evidences of dirac Large Numbers Hypothesis (LNH) have been found in the form of series of ratios relating cosmological parameters and quantum properties of space-time. A very large number on the order of $5 \times 10^{60}$ connects mass, density, age and size of the observable universe with Planck mass, density, time and length, respectively.
\end{abstract}

Keywords: Mass Relation, Fundamental Masses, Dirac Large Numbers Hypothesis, Newtonian Constant of Gravitation

\section{INTRODUCTION}

Discovery of theoretical or empirical mass relations for the many various particles is a great challenge for the recent high-energy physics and astrophysics and derivation of mass relations covering a very large range of particle masses is most desirable. Known are a few formulas connecting the masses of particles having similar properties, one such, is Hadron's multiplets (octets and decuplets of particles having close masses).

Though imprecise, one of the first attempts to empirically derive 'Balmer's law' for several particles has been attempted from (Nambu, 1952), wherein, $m_{n} \sim 137 n m_{e}$ is the mass of the $n$th particle, $m_{e}$ is mass of the electron and $n$ is an integer or half-odd. Based on SU(3) symmetry, the Gell-Mann-Okubo mass formula (Gell-Mann, 1961; Okubo, 1962) has been derived for baryon decuplet: $m_{\Delta}-m_{\Sigma}=m_{\Sigma}-m_{\Xi}=m_{\Xi}-m_{\Omega}$, where $m_{\Delta}, m_{\Sigma}$, $m_{\Xi}$ and $m_{\Omega}$ are the masses of respective hyperons. This formula successfully predicted the mass for the then undiscovered $\Omega^{-}$hyperon. The mass relations of (Georgi and Jarlskog, 1979) ensue from the SO(10) model and relate masses of charged leptons $(e, \mu$ and $\tau)$ and down-type quark $(d, s$ and $b) m_{e}=m_{d} / 3, m_{\mu}=3 m_{s}$ and $m_{\tau}=$ $m_{b}$. However, these mass relations yield results that deviate significantly as compared to experimental data. It is postulated in (Barut, 1979) that a quantized magnetic

(C) 2014 Curtis J. Forsythe and Dimitar T. Valev. This open access article is distributed under a Creative Commons Attribution (CC-BY) 3.0 license which permits unrestricted use, distribution, and reproduction in any medium, provided the original work is properly credited. DOI: 10.3844/pisp.2014.152.158 
self-energy of magnitude $3 m_{e} n^{4} /(2 \alpha)$ be added to the rest mass of a lepton to get the next heavy lepton in the chain $e, \mu, \tau, \cdots$, with $n=1$ for $\mu, n=2$ for $\tau$. Here, $\alpha$ is the fine structure constant, $\mathrm{m}_{\mathrm{e}} \approx 0.511 \mathrm{MeV}$ is the mass of the electron and $n$ is a new quantum number. Thus it was predicted $M_{\tau}=1786.08 \mathrm{MeV}$ and for the next lepton $M_{\delta}=10293.7 \mathrm{MeV}$. Koide (1993) has pointed out that the mass relation $m_{e}+m_{\mu}+m_{\tau}=(2 / 3)\left(\sqrt{m_{e}}+\sqrt{m_{\mu}}+\sqrt{m_{\tau}}\right)^{2}$ is consistent with the measurements of the tau lepton mass. Found in (Valev, 2008) is a simple mass relation $m_{i}=m_{e} \alpha_{i}(0) / \alpha$ connecting masses of stable particles (proton, electron, neutrino $v_{e}$ and graviton) with coupling constants $\alpha_{\mathrm{I}}(0)$ of the four interactions and $i=1,2,3,4$. This mass relation covers an extremely wide range of values, exceeding 40 orders of magnitude and predicts a graviton mass on the order of $10^{-69} \mathrm{~kg}$.

Found in (Forsythe, 2009) is the derived mass relation:

$M_{n}=m_{e}(\sqrt{3} \pi N)^{n} \alpha^{2 n-1}$

where, $N \sim 6.02 \times 10^{23}$ is a large pure number and $n=1,2,3,4$.

This mass formula produces four equidistant masses covering a large range of 61 orders of magnitude. Mass $M_{1} \sim 2.18 \times 10^{-8} \mathrm{~kg}$ is apparent Planck mass $m_{P}=(h c / G)^{1 / 2}, M_{2} \sim 3.80 \times 10^{12} \mathrm{~kg}$ is the apparent mass of a hypothetical quantum "Gravity Atom" whose gravitational potential is equal to electrostatic potential $e^{2} / S, M_{3} \sim 6.62 \times 10^{32} \mathrm{~kg}$ has not been identified and $M_{4} \sim$ $1.16 \times 10^{53} \mathrm{~kg}$ is the assumed proper mass of the observable universe. Now, in the present paper, we extend mass relation (1) to produce seven equidistant fundamental masses covering extremely large mass range of 122 powers of magnitude.

Dirac (1937) noticed that the ratio of the age of the universe $\mathrm{H}^{-1}$, the inverse of the Hubble parameter and the atomic unit of time, $\tau=e^{2} / m_{e} c^{3} \cong 10^{-23} s$, is a large number $N_{D} \sim 4.64 \times 10^{40}$, where e is electron charge and c is speed of light in vacuum. Additionally, the ratio of mass of the observable universe $M_{u}$ and nucleon mass is of the order of $N_{D}^{2}$ and the ratio of electrostatic $e^{2} / r^{2}$ and gravitational forces $G m_{e} / r^{2}$ between proton and electron in a hydrogen atom is $2.27 \times 10^{39}$, were $G$ is the Newtonian constant of gravitation and $m_{e}$ and $m_{p}$ are electron and proton masses respectively. These "coincidences" hint at a possible connection between macro and microphysical world known as dirac Large Numbers Hypothesis (LNH). Many other interesting ratios have been found approximately relating some astrophysical (cosmological) parameters and microscopic properties of the matter. For example Jordan (1947) noted that the mass ratio for a typical star and an electron is of the order of $10^{60}$. Narlikar (1977) shows that the ratio of the observable universe radius, $\mathrm{CH}^{-1}$ and the classical electron radius, $e^{2} / m_{e} c^{2}$ is exactly equal to $N_{D}$. Additionally, the ratio of the electron mass and Hubble mass parameter $h H / c^{2}$ is $3.39 \times 10^{38}$ (Cetto et al., 1986). Here $h=h /(2 \pi)$ is the reduced Planck constant and $H$ is the Hubble constant. Peacock (1999) points out that the ratio of Hubble distance $c H^{-1}$ and Planck length $l_{P}$ is on the order of $10^{60}$. Besides, the ratio of Planck density $\rho_{P}$ and recent critical density of the universe $\rho_{\mathrm{c}}$ is found to be on the order of $10^{121}$ (Andreev and Komberg, 2000). Further, the ratio of observable universe mass and Planck mass is on the order of $10^{61}$ (Shemi-Zadeh, 2002). These ratios between astrophysical parameters and microscopic properties of matter result mostly in large numbers that roughly agree with order of magnitude accuracy. Valev (2012) derived a series of ratios relating cosmological parameters (mass $M$, density $\bar{\rho}=\rho_{c}$, age $H^{-1}$ and size $c H^{-1}$ of the observable universe) and Planck (mass $m_{P}$, density $\rho_{P}$, time $t_{P}$ and length $l_{P}$ ) respectively, resulting in a very large number $N_{V}$, wherein $\mathrm{m}_{\mathrm{P}}$ is defined as the mass whose reduced Compton wavelength and Schwarzschild radius $r_{s}$ are equal, $l_{P}$ is identical with $r_{s}$ and $\rho_{P}$ is defined as the density of a sphere having mass $\mathrm{m}_{\mathrm{P}}$ and radius $l_{P}$ :

$$
\begin{aligned}
& \sqrt{\frac{M}{m_{H}}}=\frac{M}{m_{P}}=\frac{m_{P}}{m_{H}}=\frac{c H^{-1}}{l_{P}}=\frac{H^{-1}}{t_{P}}=\sqrt{\frac{\rho_{P}}{\bar{\rho}}}=\frac{n !}{r !(n-r) !} \\
& \sqrt{\frac{c^{5}}{2 G h H^{2}}}=N_{V} \approx 5.73 \times 10^{60}
\end{aligned}
$$

These ratios exactly connect cosmological and quantum parameters of space-time and appear to be a precise formulation and proof of LHN. In this study, we have found new evidences in support of $\mathrm{LNH}$ connecting cosmological parameters and microscopic properties of matter.

\section{EXTENDED MASS RELATION FOR SEVEN FUNDAMENTAL MASSES}

\subsection{Review of Mass Relation Concerning Four Fundamental Masses}

In the previous paper (Forsythe, 2009), Newton's law of universal gravitation is derived, based on postulated 
mass/energy resonance waves, wherein the apparent Newtonian constant of gravitation factors as:

$$
\begin{aligned}
& G=\frac{c^{3} \lambda_{\varphi}^{2}}{6 \pi h N^{2}}=\frac{h c}{6 \pi m_{\varphi}^{2} N^{2}}=\frac{\hbar c}{3\left(\pi \alpha m_{e} N\right)^{2}} \cong \\
& 6.663 \times 10^{-11} \mathrm{~m}^{3} \mathrm{~kg}^{-1} \mathrm{~s}^{-2}
\end{aligned}
$$

where, $m_{e}$ is electron rest mass, $\lambda_{\varphi}$ the resonance wavelength, $m_{\varphi}$ the associated particle mass and $N$ is a large pure number, curiously comparably with $N_{A}$, the 2006 recommended numerical value of Avogadro's number and in terms of the fine structure constant $\alpha$ and $\pi$, is shown to be given by Equation 4:

$$
N=\sqrt{8 / 3}\left(4 \pi \alpha^{5}\right)^{2} \cong 6.022 \times 10^{23}
$$

The Planck mass by convention is $m_{P}=(\hbar c / G)^{1 / 2}$ (Planck, 1959). Therefore, it follows from Equation 3 that the apparent Planck mass is given by:

$$
m_{P}=\sqrt{3} \pi \alpha m_{e} N=m_{e}(\sqrt{3} \pi N) \alpha \cong 2.18 \times 10^{-8} \mathrm{~kg}
$$

Additionally shown is that the resonance wavelength is equal to twice the first Boar orbit thus leading directly to:

$$
m_{\varphi}=\pi \alpha m_{e} \cong 2.09 \times 10^{-32} \mathrm{~kg}
$$

It is known that the fine structure constant, the coupling constant of electromagnetic interaction, i.e., a measure of its strength, is determined by the formula $\alpha=e^{2} /(\hbar c)$. Taking into consideration this formula, we find from Equation 3 that:

$$
G=\frac{e^{2}}{3 \pi^{2} \alpha^{3} m_{\mathrm{e}}^{2} N^{2}}
$$

In section II of paper (Forsythe, 2009), a hypothetical quantum "Gravity Atom" has been proposed, comprised of an electrically neutral central mass $M_{G}$ orbited by an electrically neutral particle having electron mass $m_{e}$ such that the gravitational potential $G M_{G} m_{e} / S$ is equal to an electrostatic potential $\mathrm{e}^{2} / \mathrm{S}$ and $\mathrm{S}$, the orbital radius, is a Bohr orbit. Thus, $G M_{G} m_{e}=e^{2}$, that in conjunction with Equation 7 results in:

$$
M_{G}=3 \pi^{2} \alpha^{3} m_{e} N^{2}=m_{e}(\sqrt{3} \pi N)^{2} \alpha^{3} \cong 3.80 \times 10^{12} \mathrm{~kg}
$$

It is also of interest to note that this is the mass for which the Schwarzschild radius is equal to twice the classical electron radius.

Noted in (Forsythe, 2009) is that examination of Equation 5 and 8 revels the masses $\mathrm{m}_{\mathrm{P}}$ and $M_{G}$ are members of the series suggested by Equation 1, that in conjunction with Equation 6 can also be expressed as $M_{n}=\pi^{n-1} \alpha^{2 n-2} m_{\varphi}(\sqrt{3} N)^{\mathrm{n}}$, where $n$ is the placement within the series. Employing Equation 1 and beginning at $n=1$, it is found that:

$$
\begin{aligned}
& M_{1}=m_{e}(\sqrt{3} \pi N) \alpha \cong 2.18 \times 10^{-8} \mathrm{~kg} \\
& M_{2}=m_{e}(\sqrt{3} \pi N)^{2} \alpha^{3} \cong 3.80 \times 10^{12} \mathrm{~kg} \\
& M_{3}=m_{e}(\sqrt{3} \pi N)^{3} \alpha^{5} \cong 6.63 \times 10^{32} \mathrm{~kg} \\
& M_{4}=m_{e}(\sqrt{3} \pi N)^{4} \alpha^{7} \cong 1.16 \times 10^{53} \mathrm{~kg}
\end{aligned}
$$

Identified above is the physical significance attributed to masses $M_{1}$ and $M_{2}$. Mass $M_{4}$ appears to be well within the range of estimates for the observable universe proper mass $M_{u}$ (Carvalho, 1995; Valev, 2014) and as such, it represents the upper limit of the series.

\subsection{Extended Mass Relation for Seven Fundamental Masses, a New Fundamental Constant $K$ and the Hubble Parameter}

Upon extending the series downwards to $n \leq 0$, we obtain:

$$
\begin{aligned}
& M_{0}=m_{e}(\sqrt{3} \pi N)^{0} \alpha^{-1}=\frac{m_{e}}{\alpha} \cong 1.25 \times 10^{-28} \mathrm{~kg} \\
& M_{(-1)}=m_{e}(\sqrt{3} \pi N)^{-1} \alpha^{-3}=\frac{m_{e}}{\sqrt{3} \pi N \alpha^{3}} \cong 7.15 \times 10^{-49} \mathrm{~kg} \\
& M_{(-2)}=m_{e}(\sqrt{3} \pi N)^{-2} \alpha^{-5}=\frac{m_{e}}{3 \pi^{2} N^{2} \alpha^{5}} \cong 4.10 \times 10^{-69} \mathrm{~kg}
\end{aligned}
$$

It is found that the ratio of any two consecutive masses in the Equation 9-15 is a new fundamental constant $K$, wherein:

$$
\frac{M_{n+1}}{M_{n}}=K=\sqrt{3} \pi N \alpha^{2} \cong 1.77 \times 10^{20}
$$

Therefore: 
$M_{n}=\frac{m_{e}}{\alpha}\left(\sqrt{3} \pi N \alpha^{2}\right)^{n}=\frac{m_{e}}{\alpha} K^{n}=M_{0} K^{n}$

where, $n=-2,-1,0, \ldots, 4$. We now find that:

$$
\frac{2 M_{1}}{\pi K^{3}}=\frac{2 M_{2}}{\pi K^{4}}=\frac{2 M_{3}}{\pi K^{5}}=\frac{2 M_{4}}{\pi K^{6}} \cong 2.61 \times 10^{-69} \mathrm{~kg}
$$

The current best estimates of $H_{0}$ center around about $70 \mathrm{~km} \mathrm{~s}^{-1} \mathrm{Mps}^{-1}$. Thus, when $\mathrm{m}_{\mathrm{H}}$, the Hubble mass (Maor and Brustein, 2003; Gazeau and Toppan, 2010), is defined as:

$$
m_{H}=\frac{\hbar H}{c^{2}}
$$

We find from Equation 19 an approximate value for $\mathrm{m}_{\mathrm{H}}$ of $2.66 \times 10^{-69} \mathrm{~kg}$. This result is close to that from Equation 18. It is close enough in fact that all symbolic members of Equation 18 are assumed to express accurately the value of the Hubble mass concomitant with $H$. Therefore, regarding the 1 st and 4 th members:

$$
\frac{2 m_{P}}{\pi K^{3}}=m_{H}
$$

And:

$$
\frac{2 M_{\mathrm{u}}}{\pi K^{6}}=m_{H}
$$

From which, upon elimination of $K$, results:

$$
m_{H}=\frac{2 m_{P}^{2}}{\pi M_{u}}
$$

And the final result upon substituting the right-hand member of Equation 22 into Equation 19 for $m_{H}$ and solving for $H$, becomes:

$$
H=\frac{2 c^{2}}{\pi \hbar} \frac{m_{P}^{2}}{M_{u}}=\frac{4 c^{2}}{h} \frac{m_{P}^{2}}{M_{u}}
$$

If the Hubble mass is defined as $h H / c^{2}$, as in (Forsythe, 2009), the value for $m_{H}$ would be $\sim 1.64 \times 10^{-68}$ $\mathrm{kg}$, so the left-hand members of Equation 20 and 21 must then be multiplied by $2 \pi$ to preserve the equalities and Equation 22 is still the final result.
As was proposed in (Forsythe, 2012), predicated upon the rate of cosmic expansion apparently transitioning from deceleration to acceleration at redshift $z \sim 0.5$ (Perlmutter et al., 1999), the deceleration parameter must have passed through a zero null point at transition, as the opposing operatives of cosmic expansion reached a transient state of equilibrium. Intuitively it would seem that the Hubble parameter at that juncture $H_{e q}$, the tipping point between deceleration and acceleration, must be tied to the mass of the universe via means of a unique relationship that existed at that juncture, as developed through Equation 19-22, leading to Equation 23. However, it does not necessarily follow that the Hubble parameter is increasing along with the accelerating rate of cosmic expansion. Some theoretical considerations suggest that the Hubble parameter has now assumed a truly constant value in time and space. Others predict that even as the expansion accelerates, the Hubble parameter will continue to decrease asymptotically, approaching a limiting value of about $62 \mathrm{~km} \mathrm{~s}^{-1} \mathrm{Mpc}^{-1}$, as the influence of the cosmological constant becomes more and more dominant over the contribution of matter after several billions of years and a several fold increase in the scale factor. It is thus reasonable to propose that $H_{0}$, the present day Hubble parameter and $H_{e q}$ are essentially identical. Thus Equation 24:

$$
H=H_{\mathrm{eq}}=\frac{4 c^{2}}{h} \frac{m_{\mathrm{P}}^{2}}{M_{\mathrm{u}}} \cong H_{0} \cong 68.63 \mathrm{~km} \mathrm{~s}^{-1} \mathrm{Mps}^{-1}
$$

A theoretical value for $H_{0}$ of $68.66 \pm 0.1 \mathrm{~km} \mathrm{~s}^{-1} \mathrm{Mps}^{-1}$, obtained via an entirely independent approach (Bukalov, 2002), is in excellent agreement with the above.

Since by convention, the square of the Planck mass is $h c /(2 \pi G)$ Equation 23 can be restated as:

$$
H_{e q}=\frac{2 c^{3}}{\pi G M_{u}} \cong 68.63 \mathrm{~km} \mathrm{~s}^{-1} \mathrm{Mps}^{-1}
$$

And from Equation 25, we obtain:

$$
M_{u}=\frac{2 c^{3}}{\pi G H_{e q}} \cong 1.16 \times 10^{53} \mathrm{~kg}
$$

The exact same result as that of Equation 12 . Additionally, from Equation 12 and 26 another interesting relationship results Equation 27:

$$
M_{4}=M_{u}=\frac{2 c^{3}}{\pi G H_{e q}}=m_{e}(\sqrt{3} \pi N)^{4} \alpha^{7} \cong 1.16 \times 10^{53} \mathrm{~kg}
$$




\subsection{Review of Three Fundamental Masses Obtained by Dimensional Analysis}

In previous paper (Valev, 2013), three fundamental masses have been derived by dimensional analysis, namely:

$$
\begin{aligned}
& m_{1}=\frac{\hbar H}{c^{2}}=m_{H} \\
& m_{2}=k \frac{c^{3}}{G H} \cong M_{u} \\
& m_{3}=\left(\frac{H \hbar^{3}}{G^{2}}\right)^{1 / 5} \cong 1.43 \times 10^{-20} \mathrm{~kg}
\end{aligned}
$$

In form, Equation 26 coincides closely with Equation 29 and the two are an identity when the dimensionless parameter $k$, on the order of unity, is identical with $2 / \pi$.

The papers (Forsythe, 2009; 2012) do not attribute any physical significance to mass $M_{3} \sim 6.63 \times 10^{32} \mathrm{~kg}$ in the original $n_{1}$ through $n_{4}$ series. Recently we have identified this mass with the Eddington stellar mass limit where the outward pressure of the star's radiation balances the inward gravitational force (Vink et al., 2011; Crowther et al., 2012). Additionally, we have identified the mass $M_{0} \sim 1.25 \times 10^{-28} \mathrm{~kg}$ as exactly coinciding with the mass dimension constant in a basic mass equation from paper (Valev, 2008) relating masses of stable particles and coupling constants of the four fundamental interactions. It is interesting that this mass is approximately a half-charged pion mass $M_{0}=m_{e} / \alpha \cong 0.5 m_{\pi \pm}$. Mass $M_{(-1)} \sim 7.15 \times 10^{-49} \mathrm{~kg}$ is presently unidentified and could feasibly be regarded as a prediction by the suggested model, Equation 9, for a fundamental, albeit as yet unobserved light particle. Finally, mass $M_{(-2)} \sim 4.10 \times 10^{-69} \mathrm{~kg}$ in the extended series is easily identifiable with the Hubble mass Equation 19 as $0.5 \pi m_{H}$. It is of further interest to note that the extended mass series includes seven equidistant fundamental masses covering a mass interval of 122 orders of magnitude and that masses $M_{(-2)}, M_{(-1)}$ and $M_{0}$ are particle physics masses, whereas the masses $M_{2}, M_{3}$ and $M_{4}$ describe macro objects and the Planck mass $M_{1}$ appears intermediate in relation to these two groups. In fact, it is easily shown that the Planck mass, as given by Equation 9, is the geometric mean of the extreme masses $M_{(-2)}$ and $M_{4}$ as given by Equation 15 and 12 , as is the geometric mean of masses $\mathrm{m}_{1}$ and $\mathrm{m}_{2}$ from Equation 28 and 29 when $k=1$. Valev mass $m_{3}$ from Equation 30 has not yet been identified and could be regarded as a prediction for unknown fundamental mass, most likely a yet unobserved very heavy particle.

\section{NEW EVIDENCE OF DIRAC LARGE NUMBERS HYPOTHESIS}

Recalling Equation 2 and the definition of terms therein, it is found that $N_{V} \sim 5.73 \times 10^{60}$ when the defined terms are evaluated according to: $M=c^{3} /(2 G H)$; $m_{H}=\hbar H / c^{2} ; \quad m_{P}=(\hbar c / 2 G)^{1 / 2} ; \quad 1_{P}=\left(2 G \hbar / c^{3}\right)^{1 / 2} ;$ $t_{P}=1_{P} / c=\left(2 G \hbar / c^{5}\right)^{1 / 2} ; \quad \rho_{P}=3 c^{5} /\left(16 \pi \hbar G^{2}\right)$; $\rho_{c}=3 H^{2} /(8 \pi G)$ is recent density of the universe equal to the critical one; $H^{-1}$, the age of the universe and $c H^{-1}$ is the Hubble distance.

The Equation 2 ratios appear very important because they relate cosmological parameters and the fundamental microscopic properties of matter. The Planck units imply quantization of space-time at extremely short range. Thus, the ratios represent connection between cosmological and quantum parameters of space-time and thus appear to be a precise formulation and proof of LHN. In addition, the very large number $N_{V}$ and Dirac large number $N_{D}$ (Dirac, 1937) seem connected by the approximate formula Equation 31:

$N_{D} \sim N_{V}^{2 / 3}=\left(\frac{c^{5}}{2 G \hbar H^{2}}\right)^{2 / 3} \cong 3.2 \times 10^{40}$

We now construct a similar series to (2) involving ratios of the same parameters producing the very large, number $N_{V F}$, as follows Equation 32:

$$
\begin{aligned}
& \left(\frac{2}{\pi} \frac{M_{u}}{m_{H}}\right)^{1 / 2}=\frac{M_{u}}{m_{P}}=\frac{2}{\pi} \frac{m_{P}}{m_{H}}=\frac{2}{\pi} \frac{c H^{-1}}{1_{P}}=\frac{2}{\pi} \frac{H^{-1}}{t_{P}}= \\
& \frac{2}{\pi}\left(\frac{\rho_{P}}{2 \rho_{c}}\right)^{1 / 2}=\frac{2}{\pi}\left(\frac{c^{5}}{G \hbar H^{2}}\right)^{1 / 2}=N_{V F} \cong 5.31 \times 10^{60}
\end{aligned}
$$

where, now:

$$
H=H_{e q}=2 c^{3} /\left(\pi G M_{u}\right) ; M_{u}=2 c^{3} /(\pi G H) \text { is apparent }
$$
proper mass of the universe; $m_{H}=\hbar H / c^{2}$; $m_{P}=(h c / 2 \pi G)^{1 / 2} ; \quad 1_{P}=\left(G \hbar / c^{3}\right)^{1 / 2}$; $t_{P}=1_{P} / c=\left(G \hbar / c^{5}\right)^{1 / 2} ; \quad \rho_{P}=3 m_{P} /\left(4 \pi l_{P}^{3}\right)=3 c^{5} /\left(4 \pi \hbar G^{2}\right) ;$ $\rho_{c}=3 H^{2} /(8 \pi G)$ and $G$ is according to Equation 3 . These ratios also represent a connection between cosmological and quantum parameters of space-time 
and so likewise appear to be possible new evidences of LNH. Recalling Equation 17, it is noteworthy that apparently: $N_{V F}=K^{3}=\left(\sqrt{3} \pi N \alpha^{2}\right)^{3} \cong 5.31 \times 10^{60}$ and that $N_{V F}$ and Dirac large number $N_{D}$ seem connected by the approximate formula:

$$
N_{D} \sim N_{V F}^{2 / 3}=K^{2}=\left(\sqrt{3} \pi N \alpha^{2}\right)^{2}=\left(\frac{4 c^{5}}{\pi^{2} G \hbar H^{2}}\right)^{2 / 3} \cong 3.04 \times 10^{40}
$$

Thus, by independent approaches it is apparent that we obtain very similar results, (31), (33) and (2), (32). From Equation 33, it follows that:

$$
N^{2}=\frac{N_{V F}^{2 / 3}}{3 \pi^{2} \alpha^{4}}=\frac{K^{2}}{3 \pi^{2} \alpha^{4}}
$$

That upon substitution into Equation 3 for the square of $N$ results in Equation 35:

$G=\frac{\alpha^{2} \hbar c}{m_{e}^{2} N_{V F}^{2 / 3}}=\frac{\alpha^{2} \hbar c}{m_{\mathrm{e}}^{2} K^{2}}$

Thus, Equation 34 and 35 connect $N$ to LNH and therefore to $\mathrm{G}$ through the unique and apparently new fundamental constant $K$, as given by Equation 16 .

\section{CONCLUSION}

Mass relation (1) obtained in (Forsythe, 2009) has been extended from $n=-2$ to $n=4$. The result is seven equidistant fundamental masses $M_{n}$, covering a mass interval of 122 orders of magnitude, have been obtained. Six of these masses are successfully identified, namely $M_{1} \sim 2.18 \times 10^{-8} \mathrm{~kg}$ the apparent Planck mass $m_{P}=(\hbar c / G)^{1 / 2}$, that is very important in resent particle physics. The mass $M_{2} \sim 3.80 \times 10^{12} \mathrm{~kg}$ is the central mass of a hypothetical quantum "Gravity Atom" whose gravitational potential $G M_{G} m_{e} / S$ is equal to electrostatic potential $\mathrm{e}^{2} / \mathrm{S}$ and $S$ is a Bohr orbit radius and the mass $M_{3} \sim 6.63 \times 10^{32} \mathrm{~kg}$ is of the order of the Eddington mass limit of the most massive stars. The mass $M_{4} \sim 1.16 \times 10^{53} \mathrm{~kg}$ is close to the mass of the Hubble sphere and most probably appears to be mass of the observable universe. The mass $M_{0}$ $\sim 1.25 \times 10^{-28} \mathrm{~kg}$ coincides with a mass dimension constant in a basic mass equation relating masses of stable particles and coupling constants of the four interactions; approximately a half charged pion mass. The mass $M_{(-2)} \sim 4.10 \times 10^{-69} \mathrm{~kg}$ is easily identifiable with the Hubble mass as $0.5 \pi m_{H}$. The mass $M_{(-1)}$ $\sim 7.15 \times 10^{-49} \mathrm{~kg}$ remains yet unidentified and could be regarded as a prediction by the suggested mass relation for unknown fundamental mass, most likely a yet unobserved light particle. Apparently, masses $M_{(-2)}, M_{(-1)}$ and $M_{0}$ are particle physics masses, whereas the masses $M_{2}, M_{3}$ and $M_{4}$ describe macro objects and the Planck mass $M_{1}$ appears intermediate in relation to these two groups.

Finally, new evidences of $L N H$ have been found in the form of series of ratios relating cosmological parameters and quantum properties of space-time. In addition, the very large number $N_{V F}=K^{3}=\left(\sqrt{3} \pi N \alpha^{2}\right)^{3} \cong N_{V}=\sqrt{c^{5} /\left(2 G \hbar H^{2}\right)} \cong 5.31 \times 10^{60}$ connects mass, density, age and size of the observable universe with Planck mass, density, time and length respectively and $K$ is apparently a unique and new fundamental constant.

\section{ACKNOWLEDGMENT}

We would like to thank James R. Johnson and Andrew W. Beckwith for encouraging discussions.

\section{REFERENCES}

Andreev, A.Y. and B.V. Komberg, 2000. Cosmological parameters and the large numbers of eddington and dirac. Astron. Rep., 44: 139141. DOI: $10.1134 / 1.163834$

Barut, A.O., 1979. Lepton mass formula. Phys. Rev. Lett., 43: 1057-1057. DOI: 10.1103/PhysRevLett.43.1057.2

Bukalov, A.V., 2002. Determination of the hubble parameter exact value. Phys. Consciousness Life Cosmol. Astrophys. J.

Carvalho, J.C., 1995. Derivation of the mass of the observable universe. Int. J. Theor. Phys., 34: $2507-$ 2509. DOI: $10.1007 / \mathrm{BF} 00670782$

Cetto, A., L. De La Pena and E. Santos, 1986. Dirac large-number hypothesis revised. Astron. Astrophys., 164: 1-5.

Crowther, P.A., R. Hirschi, N.R. Walborn and N. Yusof, 2012. Very massive stars and the eddington limit.

Dirac, P.A.M., 1937. The cosmological constants. Nature, 139: 323-323. DOI: 10.1038/139323a0

Forsythe, C.J., 2009. Resonance structure of matter, nature of gravitation and the quantum energy states of the hydrogen atom. Phys. Essays, 22: 112-121. DOI: $10.4006 / 1.3100617$ 
Forsythe, C.J., 2012. A transient equilibrium value for the Hubble parameter at redshift $\mathrm{z} \sim 0.5$. Phys. Essays, 25: 203-208. DOI: 10.4006/0836-139825.2.203

Gazeau, J.P. and F.A. Toppan, 2010. A natural fuzzyness of de sitter space-time. Class. Quant. Grav., 27: 025004-025004. DOI: $10.1088 / 0264-$ 9381/27/2/025004

Gell-Mann, M., 1961. The Eightfold Way: A Theory of Strong Interaction Symmetry. 1st Edn., California Inst. of Technology, Synchrotron Laboratory, Pasadena, pp: 94.

Georgi, H. and C. Jarlskog, 1979. A new lepton-quark mass relation in a unified theory. Phys. Lett. B, 86: 297-300. DOI: 10.1016/0370-2693(79)90842-6

Jordan, P., 1947. Die Herkunft der Sterne. 1st Edn., Wissenschaftliche Verlagsges, Stuttgart, pp: 71.

Koide, Y., 1993. Should the renewed tau mass value $1777 \mathrm{MeV}$ be Taken Seriously. Mod. Phys. Lett. A, 8: 2071-2078. DOI: 10.1142/S0217732393001781

Maor, I. and R. Brustein, 2003. Distinguishing among scalar field models of dark energy. Phys. Rev. D, 67: 103508-103508. 10.1103/PhysRevD.67.103508

Nambu, Y., 1952. An empirical mass spectrum of elementary particles. Prog. Theor. Phys., 7: 595-596. DOI: 10.1143/PTP.7.595

Narlikar, J.V., 1977. The Structure of the Universe. 1st Edn., Oxford University Press, London, ISBN-10: 0192890824, pp: 264.

Okubo, S., 1962. Note on unitary symmetry in strong interactions. Prog. Theoretical Phys., 27: 949-966. DOI: 10.1143/PTP.27.949
Peacock, J.A., 1999. Cosmological Physics. 1st Edn., Cambridge University Press, Cambridge, ISBN-10: 052141072X, pp: 704.

Perlmutter, S., G. Goldhaber, R.A. Knop, P. Nugent and P.G. Castro et al., 1999. Measurements of omega and lambda from 42 high-redshift supernovae. Astrophys. J., 517: 565-586. DOI: 10.1086/307221

Planck, M., 1959. The Theory of Heat Radiation. 1st Edn., Dover Publications, New York, ISBN-10: 1114813141, pp: 224.

Shemi-Zadeh, V.E., 2002. Coincidence of large numbers, exact value of cosmological parameters and their analytical representation. General Relativity Quant. Cosmol.

Valev, D., 2008. Neutrino and graviton rest mass estimations by a phenomenological approach. Aerospace Res. Bulg., 22: 68-82.

Valev, D., 2012. Derivation of three fundamental masses and large numbers hypothesis by dimensional analysis.

Valev, D., 2013. Three fundamental masses derived by dimensional analysis. Am. J. Space Sci., 1: 145-149. DOI: 10.3844/ajssp.2013.145.149

Valev, D., 2014. Estimations of total mass and energy of the observable universe. Phys. Int., 5: 15-20. DOI: 10.3844/pisp.2014.15.20

Vink, J.S., L.E. Muijres, B. Anthonisse, A. de Koter and G. Gräfener et al., 2011. Wind modelling of very massive stars up to 300 solar masses. Astron. Astrophys., 531: A132- A132. DOI: 10.1051/00046361/201116614 